\title{
Age of menarche and menstrual hygiene of pubertal female students in Bhutan: a cross sectional survey, 2017
}

\author{
Thinley Dorji ${ }^{1}$, Sangay Wangmo ${ }^{2}$, Krit Pongpirul ${ }^{3}$
}

${ }^{1}$ Kidu Medical Unit, His Majesty's Peoples 'Project, Thimphu, Bhutan

${ }^{2}$ Jigme Dorji Wangchuck National Referral Hospital, Thimphu, Bhutan

${ }^{3}$ Holistic and Oriental Medicine Research Center, Faculty of Medicine, Chulalongkorn University, Bangkok, Thailand

\begin{abstract}
Introduction: In Bhutan, pubertal and adolescent female students (10-19 years) make up $8.5 \%$ of the population. The need for menstrual hygiene management (MHM) is more urgent in post-menarcheal female students and in resource-poor settings. However, until recent time, there were strong socio-cultural barriers to discourses on menarche and MHM. This study was conducted to determine the age of menarche among Bhutanese female students to identify when to offer MHM support, their knowledge on menstruation and practices related to menstrual hygiene. Methods: This was a cross-sectional survey of female students studying in Grade 10 in Thimphu, Bhutan. A multistage clustered sampling was used. Data was collected using a self administered questionnaire developed for the purpose of this study. Results: A total of 439 post-menarcheal female students were interviewed. The mean age of menarche was $12.8+1.1$ years, and fear $(38.3 \%)$ and confusion $(25.1 \%)$ were the commonest responses. The mean score on knowledge on menstruation and MHM was 5.9+1.5 out of 9. The proportion of students with good knowledge was $36.7 \%$. The common sources of information were the mother, teacher, and sister. The majority used commercial pads $(92.5 \%)$ while some used clean cloths $(5.7 \%)$ and tampons $(1.6 \%)$. The proportion who stayed absent from school during menstruation in the month prior to data collection was $9.8 \%$. Visiting religious places $(67.6 \%)$ and participating in sports $(4.3 \%)$ were common restrictions during menstruation. Conclusions: Female students experience difficulties in menstrual hygiene management in schools. Menstruation could lead to missing schools and other activities.
\end{abstract}

Keywords: Developing country; Health behaviour; Menstrual hygiene product; Standard of living.

\section{INTRODUCTION}

Menstrual hygiene has important public health implications and is central to maternal and reproductive health in achieving the Sustainable Development Goals 3 and $6^{1,2}$. The need for menstrual hygiene management (MHM) is more urgent in post menarcheal female students and in resource-poor setting ${ }^{3,4}$. In 2017 in Bhutan, there were 67,903 female students in pubertal and adolescent age group (10-19 years), which was $8.5 \%$ of the population, that required support for $\mathrm{MHM}^{5}$.

MHM in women or adolescent female students involves the usage of a clean material to absorb or to collect menstrual blood, and this material is changed as often as necessary during the menstrual period. It includes soap and water for washing the body as required, and access to facilities to dispose off used menstrual management materials ${ }^{6,7}$.

This study aimed to assess the age of menarche among Bhutanese female students, their knowledge on menstruation and their practices related to the practice of MHM in schools.

\section{Corresponding author:}

Thinley Dorji

dorji.thinleydr@gmail.com

\section{METHODS}

\section{Study design and setting}

This cross-sectional survey was conducted in six schools in Thimphu.

\section{Study participants}

The post-menarcheal female students studying in Grade 10 in the year 2017 were included in the study. To get a representative sample for this study, a confidence interval of $95 \%$ was decided with a margin of error of 0.05 . In the absence of any sort of prior assessment in this field, variance or standard deviation was taken as 0.5 as standard practice ${ }^{8,9}$. Allowing for a $15 \%$ drop out rate, the sample size was 441 .

\section{Sampling method}

A multistage sampling was adopted. Six middle and higher secondary schools from Thimphu District (three from Thromdey and three from District) were randomly selected using lottery method from the list of sixteen schools mentioned in the Annual Education Statistics 2017 by the Ministry of Education, Bhutan. From each of the six schools, class sections from Grade 10 were 
selected randomly using lottery method until we got 24 clusters The average cluster size was 18.4 female students. Those students who had not attained menarche or absent on the days of data collection were excluded.

\section{Study tool and data collection}

A self-administered questionnaire in English was designed in consultation with a gynaecologist and a school health in-charge (teacher). The basic socio-demographic information, history on the onset of menstruation, knowledge and practices on menstruation and menstrual hygiene, and the use of absorbent (cloth, commercial pads, and tampons) were collected. The questionnaire was pre-tested in a group of 20 female students in a Middle Secondary School in Thimphu. Changes were made to the questionnaires to improve its face validity. Data was collected in June 2017

\section{Data analyses}

The data were entered into SPSS 23.0 (trial version) and cleaning done by two investigators. Data were then exported to STATA 13.1/MP (StataCorp. 2016. Stata Statistical Software: College Station, TX: StataCorp LP USA, serial number 501306208483) for analysis. Descriptive analyses were performed for each of the variables, continuous variables are presented as mean \pm standard deviation and categorical variables are presented as proportions. Knowledge was scored using nine questions - seven multiple choice questions and two fill in the blank question. The correct response for each question was given a score of 1/9. A total score of $\geq 7$ was categorized as good knowledge and the rest were scored as poor knowledge. A higher cut-off for knowledge score was taken because the questions do not have overlapping themes. The effect of age, living arrangement, parent's occupation and education, number of elder sisters on the knowledge categories were calculated using multivariable logistic regression.

\section{Ethics considerations}

Ethics approval was given by the Research Ethics Board for Health, Thimphu (REBH/Approval/2017/042 dated $12^{\text {th }}$ June 2017) and the administrative approval by the Ministry of Education and the Thimphu District Education Office. The participants were given information about the study and were invited to participate. The participants signed the informed assent form and their guardian signed the informed consent form to allow participation in this study. The female students filling up the questionnaire were seated inside the classroom one individual per table, while the male students were allowed to play outside. They were given an option to withdraw from the study if the questionnaire gave them undue stress - two female students returned the questionnaires partially filled. No personal identifiers were collected.

\section{RESULTS}

A total of 439 questionnaires were returned, constituting a response rate of $99.6 \%$. The mean age of the sample was $15.8+3.2$ years, on an average three years beyond menarche at the time of the survey.

\section{Menarcheal characteristics}

The mean age of menarche was $12.8 \pm 1.1$ years, range $10-16$ years (Figure 1). The various psychological responses to menarche were fear $(168,38.3 \%)$, confusion $(110,25.1 \%)$, feeling that menstruation was a bad thing $(79,18.0 \%)$ and anxiety $(23,5.2 \%)$. The first person to share about menarche included mother (268, $61.3 \%)$, sister $(91,20.7 \%)$, best friend $(38,8.7 \%)$, father $(17$, $3.9 \%)$ and relatives $(14,3.2 \%)$.

\section{Knowledge on menstruation}

The mean score on knowledge on menstruation and MHM was $5.9 \pm 1.5$ out of 9 . The proportion of female students with good knowledge was $36.7 \%$ (161). The details on the themes of knowledge assessed, the sources of knowledge on menstruation and MHM are shown in Table 1. Knowledge was better among those who had two elder sisters (adjusted OR $=3.0,95 \% \mathrm{CI}=$ 1.3-6.9, $p=0.009$ ).

\section{Menstrual hygiene management}

The majority $(406,92.5 \%)$ of the female students used commercial sanitary pads. Amongst them, 385 (94.8\%) bought it from within the school campus. On an average, the number of pads used per day was $3.3 \pm 1.3$. Among those who used cloths $(25,5.7 \%), 20$ students reused the cloth after washing with soap or detergent.

The majority $(400,91.1 \%)$ cleaned their external genitalia with water and soap at school during menstruation. However, almost three-quarters $(299,74.8 \%)$ did not feel comfortable cleaning at school and almost half of them (137, $45.8 \%$ ) did not have an adequate water supply. Among those who used sanitary pads and cloths, half of them $(229,53.1 \%)$ did not feel comfortable changing them at the school where half of them attributed to lack of adequate privacy to change (78, 33.3\%).

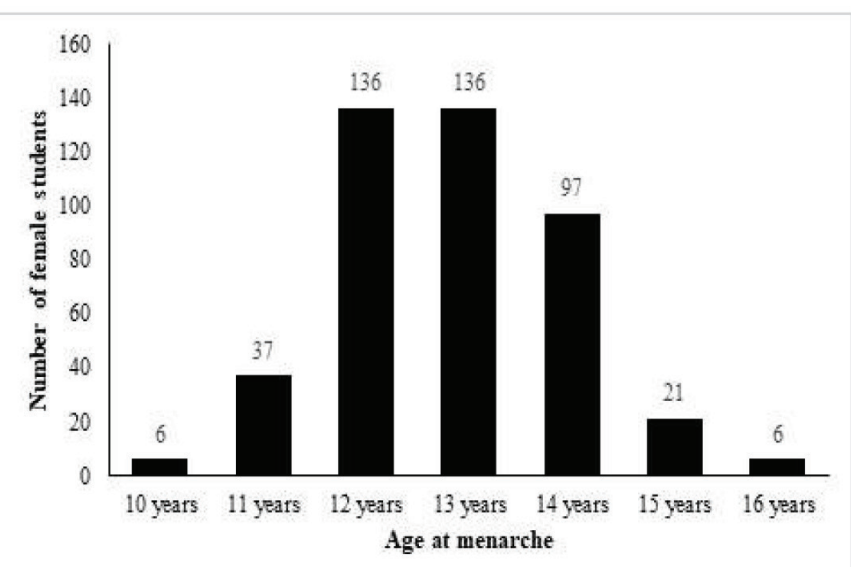

Figure 1. The age of menarche among post-menarcheal female students surveyed on menstrual hygiene management knowledge and practices in six selected schools in Thimphu, Bhutan, 2017 
Table 1. The level and sources of knowledge on menstruation and menstrual hygiene management (MHM) among post menarcheal female students in six selected schools surveyed in Thimphu, Bhutan, 2017

\begin{tabular}{|c|c|c|}
\hline Variables & $n$ & $(\%)$ \\
\hline \multicolumn{3}{|l|}{ Knowledge categories } \\
\hline Good knowledge (score 7-9) & 161 & $(36.7)$ \\
\hline Poor knowledge (score 0-6) & 278 & $(63.3)$ \\
\hline \multicolumn{3}{|l|}{ Proportion of knowledge questions with correct answers } \\
\hline Knows that menstruation is a normal event & 421 & $(96.0)$ \\
\hline Knew what is menstruation before their menarche & 368 & $(83.3)$ \\
\hline Knew the average length of a menstrual cycle ( $28-32$ days) & 230 & $(52.4)$ \\
\hline Knew the average age of menopause ( $45-55$ years) & 269 & $(61.3)$ \\
\hline Organ from where menstrual blood originates (uterus) & 84 & $(19.1)$ \\
\hline Can get pregnant after attaining menarche & 406 & $(92.5)$ \\
\hline MHM involves washing external genitalia with soap and water & 285 & $(65.0)$ \\
\hline Poor MHM leads to foul odour & 266 & $(60.6)$ \\
\hline Poor MHM leads to reproductive tract infection & 279 & $(63.6)$ \\
\hline \multicolumn{3}{|l|}{ Source of information on menstrual hygiene management* } \\
\hline Mother & 304 & $(69.3)$ \\
\hline Teacher & 168 & $(38.3)$ \\
\hline Sister & 137 & $(31.2)$ \\
\hline Friend & 93 & $(21.2)$ \\
\hline Health worker & 77 & $(17.5)$ \\
\hline Social media & 73 & $(16.6)$ \\
\hline Television & 51 & $(11.6)$ \\
\hline Books & 37 & $(8.4)$ \\
\hline
\end{tabular}

*Multiple responses were allowed

Table 2. Menstrual hygiene management and the impact of menstruation among post-menarcheal female students in six selected schools surveyed in Thimphu, Bhutan, 2017

\begin{tabular}{|c|c|c|}
\hline Variables & $n$ & $(\%)$ \\
\hline \multicolumn{3}{|l|}{ Material used for menstrual hygiene } \\
\hline Commercial sanitary pad & 406 & $(92.5)$ \\
\hline Clean cloth & 25 & $(5.7)$ \\
\hline Re-use of cloth after washing with soap or detergent & 20 & $(80)$ \\
\hline Tampons & 7 & $(1.6)$ \\
\hline None & 1 & $(0.2)$ \\
\hline Cleaning external genitalia in school & 400 & $(91.1)$ \\
\hline Access to clean water at school & 226 & $(51.5)$ \\
\hline \multicolumn{3}{|l|}{ Type of toilet in school } \\
\hline Flush type & 199 & $(45.3)$ \\
\hline Pit latrine & 240 & $(54.7)$ \\
\hline None/outdoors & o & (O) \\
\hline \multicolumn{3}{|l|}{ Disposal of soaked commercial sanitary pads* $(n=406)$} \\
\hline Dispose into dustbin & 363 & $(89.4)$ \\
\hline Throw into latrine & 12 & $(3.0)$ \\
\hline Bury & 5 & $(1.2)$ \\
\hline Take home to dispose & 44 & $(10.8)$ \\
\hline \multicolumn{3}{|l|}{ Reasons for absenteeism $*(n=43)$} \\
\hline Pain or discomfort & 21 & $(48.8)$ \\
\hline Fear of leakage & 13 & $(30.2)$ \\
\hline Feel culturally dirty & 5 & $(11.6)$ \\
\hline Shame & 4 & $(9.3)$ \\
\hline No place for disposal of pads & 4 & $(9.3)$ \\
\hline No privacy for cleaning & 3 & $(7.0)$ \\
\hline No water & 2 & $(4.6)$ \\
\hline \multicolumn{3}{|l|}{ Restrictions during menstruation* } \\
\hline Visiting religious places & 297 & $(67.6)$ \\
\hline Sports & 177 & $(40.3)$ \\
\hline Playing & 138 & $(31.4)$ \\
\hline Household works & 91 & $(20.7)$ \\
\hline Food & 73 & (16.6) \\
\hline
\end{tabular}

*Multiple responses were allowed 


\section{Impacts of menstruation}

In the last one month prior to data collection, 43 female students $(9.8 \%)$ stayed absent from school during menstruation. The mean duration of absent days was $1.3( \pm 0.6)$ days, range 1-4 days. The common symptoms and restrictions during menstruation and the reasons for absenteeism are given in Table 2.

\section{DISCUSSION}

This is the first assessment of menarche among female students in Bhutan. The age of menarche in Bhutan is similar to that of other countries in the region but lesser than that of 14.3 years reported in $2012^{11-15}$. We report on the age of menarche from interviews among post-menarcheal girls while the National Health Survey reported from women aged 10-49 years, therefore our study has lesser recall bias ${ }^{15}$

The manner in which a girl learns about menstruation and its associated changes has an impact on her response to menarche. The responses to menarche are mostly fear, anxiety, anger, shame and disgust ${ }^{16,17}$ depending on awareness and knowledge about the problem ${ }^{18,19}$.

In Bhutanese culture and the prevailing norms in the traditional system of healing (Sowa Rigpa), menarche is a sacrosanct event where the blood of menarche is considered holyzo. However, local beliefs and cultural factors have a strong influence on health perception ${ }^{21}$. Menstruation is associated with the cultural notion of "drip". Anyone coming in contact with a woman during her menstruation gets affected by drip. It is believed that a person affected by drip becomes invisible to beneficial divine forces, and thus unprotected from the destructive forces ${ }^{22}$. Therefore, there is a strong societal inhibition in talking about menstruation. The source of information on MHM in our sample like in many South Asian countries are informal, through mother, elder sisters and friends. For this to change and for fathers and male teachers to promote MHM, efforts from all sections of our society are needed.

In low-income countries, the poor knowledge on the physiology of menstruation is coupled with poor resources for MHM. In our sample, the knowledge was poor in $63 \%$ of the respondents and institutional education system to teach and promote MHM practices have started only in recent years ${ }^{23}$.

We studied MHM practices only in schools. The schools across the country are provided with toilets and water supply by the Ministry of Education and the Ministry of Works and Human Settlement. However, many female students reported that they did not feel comfortable cleaning their genitalia with soap and water in school as a part of MHM. This was due to lack of clean water and lack of adequate privacy to clean and even to dispose of their soaked pads. This highlights the need to build an environment where menstrual hygiene can be practiced with adequate privacy and dignity. It is assumed that these female students have adequate facilities for MHM at home. In 2017, there were only - 2.0\% of households that did not have a toilet facility in Bhutan; $3.0 \%$ in rural and $0.4 \%$ in urban areas according to the Population and Housing Census of Bhutan ${ }^{5}$.

We also report the common physical complaints related to menstruation. This is an important area of intervention to reduce absenteeism and restriction of activities such as playing sports doing household work. Restriction on visiting religious places in Bhutan need to be discussed in the broader scope of reducing gender disparity in health ${ }^{24}$ and promoting social inclusiveness and empowerment of women ${ }^{25}$. Perceptions and practices about menstruation changes across time and culture ${ }^{23}$ with education and wider discourse on MHM, a future study is warranted to study the trend in these restrictions.

\section{LIMITATIONS}

This sample only included female students from urban and suburban areas of Thimphu district. The situation in rural areas may be different. A qualitative approach is required to assess the psychological responses around menarche and socio-cultural barriers to discourse and interventions on MHM. We did not make physical observations of the facilities for MHM in the schools.

\section{CONCLUSIONS}

Female students experience difficulties in menstrual hygiene management in schools. Menstruation could lead to missing schools and other activities. More knowledge on menstruation and menstrual hygiene need to be given. In addition to providing facilities, schools need to work towards ensuring privacy and dignity in practising MHM.

\section{ACKNOWLEDGMENTS}

The authors would like to express their gratitude to the Ministry of Health, Ministry of Education and Thimphu District Education Office for the kind approval to conduct this study. The authors would also like to thank the school Principals, Teachers and the participants of this study, and the medical students who performed data collection. I would also like to thank Dr Mary Alice Lee for critical comments, and Saran Tenzin Tamang and Sonam Yoezer for the data collection.

\section{REFERENCES}

1. United Nations. Transforming our world: The 2030 agenda for sustainable development. 2015. [Full Text]

2. Sommer M. Menarche: A Missing Indicator in Population Health from Low-Income Countries. Public Health Rep. 2013;128(5):399-401. [Full Text]

3. Kuhlmann AS, Henry K, Wall LL. Menstrual Hygiene Management in Resource-Poor Countries. Obstet Gynecol Surv. 2017;72(6). [PubMed | Full Text | DOI] 
4. Bhattacherjee S, Ray K, Biswas R, Chakraborty M. Menstruation: Experiences of Adolescent Slum Dwelling Girls of Siliguri City, West Bengal, India. J Basic Clin Reprod Sci. 2013;2:85-91. [Full Text | DOI]

5. National Statistics Bureau. Population and Housing Census of Bhutan 2017 - National Report. Thimphu: National Statistics Bureau, Bhutan; 2018. [Full Text]

6. WASH in Schools Empowers Girls' Education: Proceedings of the $5^{\text {th }}$ Annual Virtual Conference on Menstrual Hygiene Management in Schools. New York: UNICEF; 2016. [Full Text]

7. Budhathoki SS, Bhattachan M, Castro-Sánchez E, Sagtani RA, Rayamajhi RB, Rai P, et al. Menstrual hygiene management among women and adolescent girls in the aftermath of the earthquake in Nepal. BMC Womens Health. 2018;18(1):33. [Full Text | DOI]

8. Charan J, Biswas T. How to Calculate Sample Size for Different Study Designs in Medical Research? Indian J Psychol Med. 2013;35(2):121-126. [PubMed | Full Text | DOI]

9. OpenEpi - Open Source Epidemiologic Statistics for Public Health. [Full Text]

10. Annual Education Statistics 2017. Thimphu: Ministry of Education, Royal Government of Bhutan; 2017. [Full Text]

11. Pathak PK, Tripathi N, Subramanian SV. Secular Trends in Menarcheal Age in India-Evidence from the Indian Human Development Survey. Baradaran HR, ed. PLoS One. 2014;9(11):e111027. [Full Text | DOI]

12. Sunuwar L, Saha CG, Anupa KC, Upadhyay Dhungel K. Age at menarche of subpopulation of Nepalese girls. Nepal Med Coll J. 2010;12(3):183-6. [Full Text]

13. Ghimire M, Sharma A, Ghimire M. Menarche and Its Determinants in Adolescent Girls. J Lumbini Med Coll. 2013;2(1):10-13. [Full Text | DOI]

14. Hossain MG, Islam S, Aik S, Zaman TK, Lestrel PE. Age at menarche of university students in Bangladesh: secular trends and association with adult anthropometric measures and socio-demographic factors. J Biosoc Sci. 2010;42(5):677687. [Full Text $\mid$ DOI]

15. Ministry of Health. National Health Survey 2012. Thimphu: Ministry of Health, Royal Government of Bhutan; 2012. [Full Text]
16. McPherson ME, Korfine L. Menstruation across time: menarche, menstrual attitudes, experiences, and behaviors. Womens Health Issues. 2004;14(6):193-200. [Full Text DOI]

17. Golchin NAH, Hamzehgardeshi Z, Fakhri M, Hamzehgardeshi L. The experience of puberty in Iranian adolescent girls: a qualitative content analysis. BMC Public Health. 2012;12(1):698. [Full Text | DOI]

18. Marvan ML, Alcala-Herrera V. Age at menarche, reactions to menarche and attitudes towards menstruation among Mexican adolescent girls. J Pediatr Adolesc Gynecol. 2014;27(2):61-6. [Full Text | DOI]

19. Tang CSK, Yeung DY-L, Lee AM. Psychosocial correlates of emotional responses to menarche among Chinese adolescent girls. J Adolesc Health. 2003;33(3):193-201. [Full Text | DOI]

20. Tshe-nam T. An Extensive Commentary on the Four Medical Tantras - Volume IV. Sithroen Mirig Publishers [Full Text]

21. Dorji T, Melgaard B. Medical History of Bhutan: A Chronicle of Health and Disease from Bon Times to Today. $2^{\text {nd }}$ ed. Centre for Research Initiatives; 2018. [Full Text]

22. Barth F, Wikan U. The Situation of Children in Bhutan - An Anthropological Perspective. Thimphu: Centre for Bhutan and GNH Studies; 2011. [Full Text | DOI]

23. Lahiri-Dutt K. Medicalising menstruation: a feminist critique of the political economy of menstrual hygiene management in South Asia. Gender, Place Cult. 2015;22(8):1158-1176. [Full Text | DOI]

24. The Global Gender Gap Report 2017. Geneva: World Economic Forum; 2017. [Full Text]

25. BHUTAN Gender Equality Diagnostic of Selected Sectors. Philippines: Asian Development Bank; 2014. [Full Text] 